\title{
A promising CpG adjuvant-loaded nanoparticle-based vaccine for treatment of dust mite allergies
}

\author{
"The development of such a vaccine is important because in the USA, \\ $84 \%$ of homes have measurable levels of house dust mite allergens \\ and a quarter of these houses have greater levels of allergens than \\ the proposed limit for asthma."
}

\begin{abstract}
Keywords: allergy $\bullet$ asthma $\bullet$ biodegradable $\bullet$ CpG $\bullet$ Der p2 $\bullet$ house dust mite allergens - nanoparticles • PLGA
\end{abstract}

We have recently reported on the development of a nanoparticle-based vaccine for protection and treatment against dust mite allergies [1]. The development of such a vaccine is important because in the USA, 84\% of homes have measurable levels of house dust mite (HDM) allergens and a quarter of these houses have greater levels of allergens than the proposed limit for asthma [2]. Continuous exposure to HDM allergens can lead to lung inflammation as evidenced by lung eosinophilia and airway obstructions. This can lead to asthma attacks in sensitized individuals. Persistent exposure to HDM allergens stimulates dendritic cells (DCs) in lungs to initiate T-helper 2 (Th2) immune responses [3]. These Th2 responses promote eosinophil recruitment to lungs, inflammatory cytokines; and $\mathrm{B}$ cell production of immunoglobulin (Ig) E antibodies [4]. Existing strategies for treatment of the symptoms of dust mite allergies include the use of $\beta 2$ agonists and neutralizing antibodies such as Xolair ${ }^{\circledR}$ (omalizumab). Subcutaneous or sublingual immune-based therapies have been developed and tested to generate HDM-specific long-term tolerance, and have shown promising results in adults and children. However, a drawback to such approaches are that they require regular treatments of high doses of HDM allergens for at least 3 years to show acceptable clinical efficacy. As such, there is a critical need for a longterm solution to HDM-induced asthma. The increased levels of HDM allergens present in households creates a need for the development of a vaccine that can switch the inflammatory immune response induced by HDM allergens to a protective immune response. This vaccine should ideally induce T-helper 1 (Th1) immune responses that result in production of IFN- $\gamma$ and IgG2a antibodies [5]. The vaccine would also ideally reduce Th2-stimulated airway inflammation and initiate protective immunity by Th1 cells that would reduce symptoms associated with the allergy.

Dermatophagoides pteronyssinus (Der p) is one of the most common allergy-causing mites and produces Der p2, which is a strong immunogenic allergen [6]. Serum samples of $79 \%$ of patients suffering from asthma, rhinitis and/or wheezing test positive for titers of anti-Der $\mathrm{p} 2 \mathrm{IgE}$ antibodies, making it a strong potential allergen for development of vaccines against $\mathrm{HMD}$ allergies [7]. However, subcutaneous injections of Der p2 allergen alone to mice caused adverse increases in the production of IgE antibodies and Th2-biased immune responses [8]. This resulted in the need for a vaccine formulation that allowed for co-delivery of the allergen with adjuvants
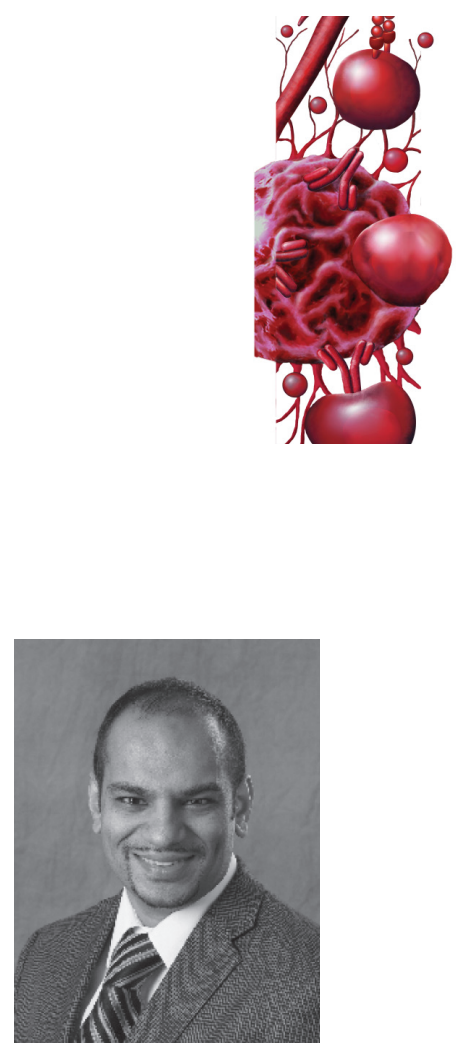

Aliasger K Salem Department of Pharmaceutical Sciences \& Experimental Therapeutics, College of Pharmacy, University of lowa, lowa City, IA 52242, USA aliasger-salem@uiowa.edu 
that promote Th1-biased allergen specific immune responses to reduce lung damage linked with exposure to allergen [9]. Unmethylated cytosine-phosphateguanine $(\mathrm{CpG})$ oligonucleotides are used as a potent adjuvant for shifting immune responses to the Th1 type [10,11]. It is a Toll-like receptor-nine agonist which activates DCs and B cells to produce Th1-type cytokines and suppresses Th2-triggered allergic responses [9]. Co-administration and/or chemical conjugation of $\mathrm{CpG}$ with HDM allergen has been shown to decrease eosinophilia and IL-5 production while increasing the production of IFN- $\gamma$ [12]. Peripheral DCs isolated from healthy patients vaccinated with ragweed allergen conjugated to $\mathrm{CpG}$ induced increased levels of Th1 cytokines and decreased levels of Th2 cytokines [13]. Chemical conjugation of $\mathrm{CpG}$ with allergen does have some limitations. It is expensive and can lead to structural alteration of fused molecules changing their immunostimulatory properties. Furthermore, in vivo cleavage of the bridge linking the allergen and adjuvant can prevent co-delivery of molecules to the same DCs.

c6

"...biodegradable nanoparticle-based vaccination strategy has significant potential for treating

house dust mite allergies and is ripe for further development.

An alternative co-delivery method is to vaccinate with CpG and Der p2-loaded biodegradable poly(lacticco-glycolic acid) (PLGA) polymer particles. In addition to co-delivering multiple molecules, PLGA particlebased vaccines can stimulate strong Th1 type responses as determined by measured levels of IgG2a antibodies. We have shown that PLGA particles loaded with antigen and $\mathrm{CpG}$ can stimulate potent antigen-specific immune responses when compared with vaccination of antigen and CpG in solution $[14,15]$. We have also shown that biodegradable particles loaded with CpG and antigen generate stronger antigen-specific IgG2a antibody responses than $\mathrm{CpG}$-antigen conjugates at later time points. This is presumably because of the sustained release characteristics of the particle-based vaccine [16]. In addition, we have shown that the magnitude of immune response generated is correlated with the size of PLGA particles used for immunization [15]. While large particles loaded with antigen and $\mathrm{CpG}$ are known to produce high levels of total IgG1 antibodies, nanoparticles containing antigen with $\mathrm{CpG}$ have been shown to induce higher ratios of IgG2a to IgG1 antibodies. To develop a vaccine against allergy-associated lung disorders, stimulation of high IgG antibodies and Th1 type immune responses is necessary. Th1-based immunity would be expected to decrease the secretion of $\operatorname{IgE}$ antibody and inflammatory damage to lungs follow- ing exposure to allergen. We have recently shown that PLGA nanoparticles that co-deliver CpG and Der p2 can generate increased Der p2 specific IgG2a antibody responses and decreased Der p2 specific IgE antibody responses. In these studies, we carefully characterized the effects of the size of PLGA particle vaccines and the influence of $\mathrm{CpG}$ in the overall immune response to Der p2-coated PLGA particle vaccines [1]. We showed that the size of the PLGA particle and the presence of CpG had a significant impact on the extent and type of immune response stimulated against Der $\mathrm{p} 2$ antigen. Three different sizes of CpG-loaded PLGA particles were prepared using a double emulsion solvent evaporation and differential centrifugation method. Mice were vaccinated with Der $\mathrm{p} 2$ and the different sizes of blank or CpG-loaded PLGA particles. The vaccinated mice were then exposed to intranasal instillation of Der p2 daily for 10 days. This was followed by euthanization to determine leukocyte accumulation in bronchoalveolar lavage (BAL) fluids, antibody response profiles and airway hyper-responsiveness. Vaccination with the PLGA particles resulted in a size-dependent decrease in the level of eosinophils found in BAL fluids. Mice vaccinated with nine micron-sized blank PLGA particles coated with Der p2 showed increased levels of IgE and IgG1 antibodies, as well as increased airway hyperresponsiveness. Loading CpG into PLGA particles of all sizes prevented airway hyper-responsiveness after Der p2 exposures. Inflammatory responses to Der p2 exposure were significantly reduced when smaller PLGA particles were used for vaccination. In addition, loading CpG in PLGA particles increased Der p2-specific IgG2a antibody levels. Our studies have shown that the size of PLGA particles used for vaccination plays a significant role in the prevention of HDM-induced allergy and that incorporation of $\mathrm{CpG}$ into the PLGA particles preferentially develops a Th1-type immune response. This biodegradable nanoparticle-based vaccination strategy has significant potential for treating HDM allergies and is ripe for further development. Future studies will need to evaluate the potential of the vaccine in a therapeutic model of pre-existing allergy and will need to identify the optimal dose concentrations, dose intervals and number of doses for generating maximum therapeutic efficacy. In addition to control over size [15], biodegradable nanoparticles are amenable to further manipulations such as changing surface chemistries [17], conjugating cell binding ligands, changing the polymer used [18] and packaging multiple synergistic adjuvants into the nanoparticles to further enhance the therapeutic response. The particles can also be used as a prime to alternative vaccination strategies or combined with small-molecule-based therapies to generate stronger therapeutic outcomes $[19,20]$. 


\section{Financial \& competing interests disclosure}

The author gratefully acknowledges support from the National Institute of Environmental Health Sciences-funded Environmental Health Sciences Research Center (NIH P30 ES005605). Other sources of support include the American Cancer Society (RSG-09-015-01-CDD), the National Cancer Institute (NIH 1R21CA13345-01/1R21CA128414-01A2/UI Mayo Clinic Lym- phoma SPORE) and the Lyle and Sharon Bighley Professorship. The author has no other relevant affiliations or financial involvement with any organization or entity with a financial interest in or financial conflict with the subject matter or materials discussed in the manuscript apart from those disclosed.

No writing assistance was utilized in the production of this manuscript.

\section{References}

1 Joshi VB, Adamcakova-Dodd A, Jing X et al. Development of a Poly (lactic-co-glycolic acid) Particle Vaccine to Protect Against House Dust Mite Induced Allergy. AAPS J. doi:10.1208/s12248-014-9624-5 (2014)(Epub ahead of print).

2 Arbes SJ Jr, Cohn RD, Yin M et al. House dust mite allergen in US beds: results from the First National Survey of Lead and Allergens in Housing. J. Allergy Clin. Immunol. 111(2), 408-414 (2003).

3 Lambrecht BN, Hammad $\mathrm{H}$. The airway epithelium in asthma. Nat. Med. 18(5), 684-692 (2012).

4 Johnson JR, Wiley RE, Fattouh R et al. Continuous exposure to house dust mite elicits chronic airway inflammation and structural remodeling. Am. J. Resp. Crit. Care 169(3), 378-385 (2004).

5 Mazzarella G, Bianco A, Catena E, De Palma R, Abbate GF. Th1/Th2 lymphocyte polarization in asthma. Allergy 55, 6-9 (2000).

6 Kidon MI, Chiang WC, Liew WK et al. Mite componentspecific IgE repertoire and phenotypes of allergic disease in childhood: the tropical perspective. Pediatr. Allergy Immunol. 22(2), 202-210 (2011).

7 Trombone APF, Tobias KRC, Ferriani VPL et al. Use of a chimeric ELISA to investigate immunoglobulin E antibody responses to Der p 1 and Der p 2 in mite-allergic patients with asthma, wheezing and/or rhinitis. Clin. Exp. Allergy 32(9), 1323-1328 (2002).

8 Tan LK, Huang CH, Kuo IC, Liew LM, Chua KY. Intramuscular immunization with DNA construct containing Der p 2 and signal peptide sequences primed strong IgE production. Vaccine 24(29-30), 5762-5771 (2006).

9 Fonseca DE, Kline JN. Use of CpG oligonucleotides in treatment of asthma and allergic disease. Adv. Drug Deliver. Rev. 61(3), 256-262 (2009).

10 Geary SM, Lemke CD, Lubaroff DM, Salem AK. Tumor immunotherapy using adenovirus vaccines in combination with intratumoral doses of CpG ODN. Cancer Immunol. Immunother. 60(9), 1309-1317 (2011).
11 Krishnamachari Y, Salem AK. Innovative strategies for co-delivering antigens and $\mathrm{CpG}$ oligonucleotides. Adv. Drug Deliver. Rev 61(3), 205-217 (2009).

12 Mo JH, Park SW, Rhee CS et al. Suppression of allergic response by $\mathrm{CpG}$ motif oligodeoxynucleotide-house-dust mite conjugate in animal model of allergic rhinitis. $\mathrm{Am}$. J. Rhinol. 20(2), 212-218 (2006).

13 Simons FE, Shikishima Y, Van Nest G, Eiden JJ, Hayglass KT. Selective immune redirection in humans with ragweed allergy by injecting Amb a 1 linked to immunostimulatory DNA. J. Allergy Clin. Immunol. 113(6), 1144-1151 (2004).

14 Zhang XQ, Dahle CE, Baman NK, Rich N, Weiner GJ, Salem AK. Potent antigen-specific immune responses stimulated by codelivery of CpG ODN and antigens in degradable microparticles. J. Immunother. 30(5), 469-478 (2007).

15 Joshi VB, Geary SM, Salem AK. Biodegradable particles as vaccine delivery systems: size matters. AAPS J. 15(1), 85-94 (2013).

16 Zhang XQ, Dahle CE, Weiner GJ, Salem AK. A comparative study of the antigen-specific immune response induced by co-delivery of $\mathrm{CpG}$ ODN and antigen using fusion molecules or biodegradable microparticles. J. Pharm. Sci. 96(12), 3283-3292 (2007).

17 Zhang XQ, Intra J, Salem AK. Conjugation of polyamidoamine dendrimers on biodegradable microparticles for nonviral gene delivery. Bioconjug. Chem. 18(6), 2068-2076 (2007).

18 Joshi VB, Geary SM, Carrillo-Conde BR, Narasimhan B, Salem AK. Characterizing the antitumor response in mice treated with antigen-loaded polyanhydride microparticles. Acta biomater. 9(3), 5583-5589 (2013).

19 Lemke CD, Geary SM, Joshi VB, Salem AK. Antigencoated poly alpha-hydroxy acid based microparticles for heterologous prime-boost adenovirus based vaccinations. Biomaterials 34(10), 2524-2529 (2013).

20 Goforth R, Salem AK, Zhu X et al. Immune stimulatory antigen loaded particles combined with depletion of regulatory T-cells induce potent tumor specific immunity in a mouse model of melanoma. Cancer Immunol. Immunother. 58(4), 517-530 (2009). 